\title{
Assessment of Factors Influencing Adherence to Antidiabetic Drugs Among Patients with Type 2 Diabetes Mellitus at a Tertiary Care Hospital in India
}

\author{
Nandini HC, Akshay Gali, Sushma Muraraiah \\ Department of Pharmacology, \\ Bangalore Medical College and Research Institute, Bangalore, Karnataka, India.
}

\begin{abstract}
Diabetes mellitus is a chronic endocrine disorder that requires long-term treatment. Non-adherence to anti-diabetic drugs is common and associated with poor outcomes. Non-adherence leads to complications, higher incidence of hospital admissions and imposes economic burden. Hence, the present study was undertaken to measure adherence to anti-diabetic medication and factors contributing to it. This cross-sectional study was conducted among patients on anti-diabetic medication after taking their informed consent in a tertiary care hospital in India. Each patient's demographic, clinical, and treatment data along with the adherence to treatment has been assessed during a personal interview with each patient using Medication Adherence Report Scale (MARS-5). Factors contributing to medication adherence was assessed using chi-square test. A total of 250 patients were recruited in the study. The mean age was 54 years and non-adherence to anti-diabetic medications was 21.6\%. Univariate Analysis showed that marital status, education status, good glycemic control, illness $>5$ years had a statistically significant association $(p=<0.05)$ with self-reported adherence to anti-diabetic medication.
\end{abstract}

Keywords : diabetes mellitus, medication adherence, anti-diabetic drugs

\section{Introduction}

Diabetes mellitus (DM) is a group of metabolic disorders characterized by hyperglycemia resulting from defects in insulin secretion, insulin action or both. ${ }^{1}$ Type $2 \mathrm{DM}$ is one of the fastest growing health challenges of the 21 st century and International Diabetes Federation estimates that about 463 million adults are living with diabetes worldwide.

India stands second in the world next to China in terms of prevalence of DM with 77 million living with DM in 2019, which is expected to raise to 101 million by 2030 .
In 2017, globally deaths due to diabetes was estimated to be 4 million. ${ }^{2}$

Adequate management of hyperglycemia in DM is important to prevent or delay complications and improve quality of life. Treatment strategies include lifestyle management, oral anti-diabetic drugs or insulin. Long-term maintenance therapy is recommended for all the patients. However, non-adherence to anti-diabetic medications is prevalent that ranges from 36 to $93 \%$ globally. ${ }^{3}$ Poor adherence to medications is one of the important causes of sub-optimally

Corresponding author: Sushma Muraraiah. Department of Pharmacology, Bangalore Medical College and Research Institute, Bengalore, Karnataka, India. Email: sushmamurari@yahoo.co.in.

Received: 29 February 2020. Revised: 27 March 2020. Published: 1 April 2020. 
controlled DM which results in accelerated development of macro and micro-vascular complications, thereby increase in mortality rate. $^{4}$

The problem of non-adherence to medication is prevalent across various chronic medical conditions and has not spared DM. Many studies have identified various factors contributing to non-adherence to anti-diabetic medication which includes younger age, ethnicity, lower socio-economic status, lower education level and complex regimens. ${ }^{4}$ Such studies provide insight into the factors that are contributing to non-adherence to medication. They will also direct towards framing preventive interventions, so that the optimum therapeutic outcome can be achieved.

Non-adherence to anti-diabetic medication in India ranges from $45 \%$ to $72 \% .{ }^{5,6,7}$ As the prevalence of DM is constantly increasing in India, addressing the issue of non-adherence is of prime importance to reduce morbidity and economic burden. In this regard, the present study was undertaken to measure adherence to anti-diabetic medications and factors contributing to the same. The results of the present study would indicate the predictors of non-adherence that would help in framing prevention strategies.

\section{Methods}

The study was conducted after obtaining approval from the Institutional Ethics Committee of Bangalore Medical College and Research Institute. Informed consent was obtained from each patient before the study.

This analytical cross-sectional study was conducted over a period of 3 months from September to November 2018, at Diabetic Clinic in Department of Medicine of Victoria Hospital, attached to Bangalore Medical College And Research Institute, Bengalore, which works on Tuesdays and Thursdays every week.

Based on previous study by Kavitha et al, non-adherence to anti-diabetic medication was noted in $28 \%$ of diabetic patients. ${ }^{6}$ The sample size was calculated considering prevalence as $28 \%$, relative precision of $20 \%$ and confidence interval $95 \%$ as 250 .

Patients diagnosed with Type 2 DM attending Diabetic Clinic being treated with anti-diabetic medications at least for 3 months and who provide Informed Consent were included in the study. Patients were selected as per computer generated random numbers. Demographic, clinical and treatment related data was recorded in the study proforma. Patients taking more than 1 anti-diabetic medication were said to be receiving polytherapy. Patients with HBA1c $\leq 7$ is considered to be under good glycemic control. Their adherence was assessed using Medication Adherence Report (MARS-5) scale. The influence of demographic and clinical variables on self-reported adherence was evaluated.

Medication adherence report scale (MARS-5) are:

1. I forgot to take my diabetes medicines

2. I altered the dose of my diabetes medicines

3. I stopped taking my diabetes medicine for a while

4. I decided to miss out a dose of my diabetes medicine

5. I took less diabetes medicine than instructed

Patients answer each question with:

Always $=1$ point

Often $=2$ points

Sometimes $=3$ points

Rarely $=4$ points; Never $=5$ points.

Score of 25 is taken as adherent to medication 


\section{Statistical Analysis}

Data were analyzed using Microsoft Excel sheet and Vassar Stats. The data collected were subjected to descriptive analysis to evaluate the adherence to anti-diabetic medications. Results were expressed as percentages, as mean \pm standard deviation for continuous parametric variables. Comparisons between the adherent and non-adherent groups were performed using Chi-square test was applied to test the association between groups. A $\mathrm{p}$-value $<0.05$ is considered statistically significant.

\section{Results and Discussion}

Medication adherence is the most important part of chronic disease management. ${ }^{8}$ Adherence is concerned about the extent to which the patient achieves an agreed upon treatment without close supervision. ${ }^{9}$ Low adherence to prescribed medical interventions is a complex problem, especially for patients with a chronic illness because of its association with increased emergency room visits, hospitalizations and suboptimal clinical outcomes, which in turn leads to increased burden on the health care system. ${ }^{10}$

Non adherent diabetic patients are at increased risk of development of micro and macro vascular diseases, hospitalizations and death. Yet, many patients fail to adhere to treatment recommendations. ${ }^{9}$ Hence, the present study was undertaken to assess the extent and factors influencing adherence behaviour to anti diabetic medications in our centre, so that preventive strategies can be framed.

Total of 250 patients were included in the study. Mean age of the patients was 54 years and included 121 males and 129 female (table 1). 200 patients were married and 50 were either single or separated. 179 of them had polytherapy and 71 had monotherapy. $78.4 \%$ of the patients were adherent to antidiabetics and $21.6 \%$ were non-adherent (Table 1).

Non adherence to anti diabetic medications was seen among $21.6 \%$ of the study patients. Dimatteo MR's quantitative review on studies regarding medication adherence over 50 years, concluded that average adherence to medications among the patients with chronic disease was $76.3 \%$ and of diabetic patients was $67.5 \%{ }^{11}$ In the present study, adherence to anti diabetic medications was $78.4 \%$. The higher frequency of adherence noted in the present study could be due to various reasons. The assessment of adherence in the present study was subjective based on patient recall about medication intake. The study was conducted in a single centre and study participants were from diabetic speciality clinic, where regular medication counselling is provided. The patients receive free anti diabetic medication in the study centre which reduces the cost related non adherence.

We noted a significant association between level of education and medication adherence, which was consistent with other studies. ${ }^{11,12,13 \mathrm{~A}}$ qualitative review by Jin et al, has quoted that education level may or may not be associated with adherence to medication. Educated patients are more likely to know the consequences of non-adherence to medication and the associated complications of diabetes. ${ }^{14}$ Hence, may tend to adhere to their medications. However, it has been also proposed that patients with lower educational level might have more trust in physicians advice and be adherent to medications. ${ }^{8}$

This study noted that T2DM patients with longer duration of illness ( $>5$ years) were more adherent which was similar to other studies. ${ }^{15,16,17 \mathrm{~A}}$ study conducted in Uganda reported that patients with DM for $>3$ years were adherent to medication and the author 
Table 1. Characteristics of the Patients Assessed for Adherence to Anti-diabetic Medications (n= 250)

\begin{tabular}{lcc}
\hline Variable A & $\begin{array}{l}\text { Number of pa- } \\
\text { tients }\end{array}$ & Percentage (\%) \\
\hline Age in years (mean) & $54.14 \pm 6.97$ & \\
\hline Gender & 121 & 48 \\
1. Male & 129 & 51.6 \\
2. Female & & \\
\hline Education Status & 37 & 14.8 \\
1. Uneducated & 213 & 85.2 \\
2. Educated & & \\
\hline Marrital Status & 200 & 80 \\
1. Married & 50 & 20 \\
2. Unmarried & 138 & 55.2 \\
\hline Residence & 112 & 44.8 \\
1. Urban & & \\
2. Rural & 66 & \\
\hline Family history (type 2 DM) & 184 & 73.6 \\
1. Yes & & \\
2. No & 196 & 21.6 \\
\hline Adherence to anti diabetic medica- & 54 & \\
tions & & \\
1. Adherent & $4.75+/-1.52$ years & \\
2. Non adherent & & \\
\hline Duration of treatment in years & & \\
\hline
\end{tabular}

also opined that patients with longer duration of illness will have frequent visits to health care professionals which may improve their understanding about the disease that in-turn improves adherence. ${ }^{16}$

As shown in Table 2 patients who were married were significantly adherent to anti diabetic medication as compared to unmarried patients $(\mathrm{p}=0.025)$. Patients with DM $>5$ years were significantly adherent to anti diabetic medication as compared to patients who had DM for $<5$ years $(p=0.0056)$. Patients who had achieved target glycaemic control (HbA1c <7) were significantly adherent to anti diabetic medication as compared to patients who had not achieved target glycaemic control (0.0013). Educated patients were significantly adherent to anti diabetic medication as compared to uneducated patients $(\mathrm{p}=0.0005)$.

Moreover, $28 \%$ of the study population were on monotherapy, while $72 \%$ were on multiple drugs. Among this, $23 \%$ on monotherapy and $55 \%$ on polytherapy were adherent to medications. In the meta analysis ${ }^{17}$ by Claxton AJ et al on associations of dose regimens with medication adherence reported that medication adherence to single dose was $79 \%$ which reduced to $51 \%$ with 4 doses. This indicates $10 \%$ decrease in medication adherence with each additional daily dose.

In contrast, Kirkman et $\mathrm{al}^{18}$ studied the adherence pattern of $>200,000$ patients on 
Table 2.Comparison of Characteristics of Patients Assessed for Adherence to Anti-diabetic Medications $(n=250)$

\begin{tabular}{|c|c|c|c|}
\hline Characteristics & $\begin{array}{c}\begin{array}{c}\text { Adherence } \\
(n=196)\end{array} \\
\end{array}$ & $\begin{array}{l}\text { Non-Adher- } \\
\text { ence }(n=54)\end{array}$ & p value \\
\hline \multicolumn{4}{|l|}{ Age } \\
\hline 1. $<50$ years & 49 & 18 & 0.220 \\
\hline 2. $>50$ years & 147 & 36 & \\
\hline \multicolumn{4}{|l|}{ Gender } \\
\hline 1. Male & 98 & 23 & 0.334 \\
\hline 2. Female & 98 & 31 & \\
\hline \multicolumn{4}{|l|}{ Residence } \\
\hline 1. Urban & 112 & 26 & 0.238 \\
\hline 2. Rural & 84 & 28 & \\
\hline \multicolumn{4}{|l|}{ Marrital Status } \\
\hline 1. Married & 151 & 49 & $0.025 *$ \\
\hline 2. Unmarried & 45 & 5 & \\
\hline \multicolumn{4}{|c|}{ Duration of Diabetes } \\
\hline 1. $<5$ years & 89 & 36 & $0.0056^{*}$ \\
\hline 2. $>5$ years & 107 & 18 & \\
\hline \multicolumn{4}{|l|}{ Therapy\# } \\
\hline 1. Monotherapy & 58 & 13 & 0.42 \\
\hline 2. Polytherapy & 138 & 41 & \\
\hline \multicolumn{4}{|l|}{ Gylcemic Control } \\
\hline 1. No & 87 & 11 & $0.0013^{*}$ \\
\hline 2. Yes & 109 & 43 & \\
\hline \multicolumn{4}{|l|}{ Education Status } \\
\hline 1. Educated & 175 & 38 & $0.0005^{*}$ \\
\hline 2. Uneducated & 21 & 16 & \\
\hline
\end{tabular}

anti-diabetic medication, reported that total daily pill burden was positively associated with medication adherence. For every additional pill taken per day, the medication adherence increased by $22 \%$. Our study also showed similar results, where patients on multiple drugs were more adherent which could be due to better medication counselling and free medications.

Majority of the patients with good glycaemic control were significantly adherent to medications. It is self-explanatory that being adherent to the drug regimens will improve glycaemic control. The association of adherence with glycaemic control has been established by many studies., ${ }^{49}$ However, apart from medication adherence, many other factors like diet, exercise, etc also are known to influence glycaemic control of the patients.

This study shows that there was significant adherence to medications among married people. Marital status has positive influence on the medication adherence because of the help and support from the spouse. However, association between the marital status 
and medication adherence is found to be controversial. Jin et al has reported that the association of marital status and medication adherence is disease specific. ${ }^{14}$

Age was not identified as a contributing factor for adherence in the present study. But generally other studies have reported younger age to be less adherent to medication. ${ }^{20}$ In the present study, we also noted the same trend that patients more than 50years were more adherent, though the results were no it statistically significant. Gender, place of residence and number of drugs did not influence the adherence behaviour of the patients.

The study had few limitations. It was based on the self-report assessed by a questionnaire, which could be over/under estimation of the adherence to medication. Generalisability of the results is limited as it was conducted in a single centre and in a specialty clinic.

\section{Conclusion}

In the present study, $78.4 \%$ were of the patients were found to be adherent to antidiabetic medication. Patients with higher education level, glycaemic control, duration of illness for more than $>5$ years and married patients were found to be significantly adherent to anti-diabetic medication. There is a need in our hospital to frame preventive strategies that focuses more on patients with lower education level, unmarried patients as well as newly diagnosed uncontrolled diabetic patients. Regular medication counselling and improving interaction between doctor and patient may improve adherence to antidiabetic patients.

\section{Acknowledgment}

The authors would like to thank all the faculty members of the Department of Pharmacology, Bangalore Medical College and Research
Institute, Bangalore, for their support in conducting the study

\section{Funding}

None

\section{Conflict of Interest}

None.

\section{References}

1. American Diabetes Association. Improving care and promoting health in populations: Standards of Medical Care in Diabetes-2020. Diabetes Care. 2020 jan;43(Suppl.1): S7-S13.

2. Saeedi P, Petersohn I, Salpea P, Malanda B, Karuranga S, Unwin N et al. Global and regional diabetes prevalence estimates for 2019 and projections for 2030 and 2045: Results from IDF Diabetes Atlas, 9th edition. Diabetes Research and Clinical Practice. 2019 Nov;157:107843

3. Arifulla M, John LJ, Sreedharan J, Muttappallymyalil J, Basha SA. Patients' Adherence to Anti-Diabetic Medications in a Hospital at Ajman, UAE. Malaysian Journal of Medical Science. Jan-Feb 2014; 21(1): 44-49

4. Sapkota S, Brien J-a, Greenfield J, Aslani P. A Systematic Review of Interventions Addressing Adherence to Anti-Diabetic Medications in Patients with Type 2 Diabetes-Impact on Adherence. PLoS One. feb2015;10(2):e0118296. doi:10.1371/ journal.pone. 0118296

5. Venkatesan M, Dongre AR, Ganapathy K. A Community-Based Study on Diabetes Medication Nonadherence and its Risk Factors in Rural Tamil Nadu. Indian Journal of Community Medicine. 2018 Apr-Jun;43(2):72-76

6. Kavitha S, Nalini GK, Suresh RM, Sahana GN, Deepak P, Nagaral JV. Treatment adherence and factors contributing to non adherence among type 2 diabetes mellitus 
patients in a tertiary care hospital: a cross sectional study. International Journal of Basic Clinical Pharmacology. 2017;6:68994.

7. Shrestha SS, Shakya R, Karmacharya BM, Thapa P. Medication adherence to oral hypoglycemic agents amongst type II diabetic patients and their clinical outcomes with special reference to fasting blood glucose and glycosylated hemoglobin levels. Kathmandu University Medical Journal.2013;11:226-32.

8. Shaimol T, Biji CR, Anilasree BP, Jayakrishnan SS, Babu G. Medication Adherence to Oral Hypoglycemic Agents in Type 2 Diabetic Patients. Journal of Pharmaceutical Research and Clinical Practice, April-June 2014; 4(2):8-12

9. Vermeire E, Hearnshaw H, Van Royen P, Denekens J. Patient adherence to treatment: three decades of research. A comprehensive review. Journal of Clinical Pharmology Therapy. 2001;26(5):331342.

10. Skinner TC, John M, Hampson SE. Social support and personal models of diabetes as predictors of self-care and well-being: a longitudinal study of adolescents with diabetes. Journal of Pediatric Psychology. 2000 Jun;25(4):257-67

11. DiMatteo MR. Variations in patients' adherence to medical recommendations: quantitative review of 50 years of research. Medical Care 2004; 42: 200-9.

12. Bruce SP, Acheampong F, Kretchy I. Adherence to oral anti-diabetic drugs among patients attending a Ghanaian teaching hospital. Pharmacy Practice. 2015 Jan-Mar;13(1):533.

13. Lemay J, Waheedi M, Al-Sharqawi S, Bayoud T. Medication adherence in chronic illness: do beliefs about medications play a role?. Patient Prefer Adherence.2018:12 1687-169

14. Jin J, Sklar GE, Sen Oh VM, Chuen
Li S. Factors affecting therapeutic compliance: A review from the patient's perspective. Therapeutic and Clinical Risk Management. 2008;4(1):269-86.

15. Abebaw M, Messele A, Hailu M, Zewdu F. Adherence and Associated Factors towards Antidiabetic Medication among Type II Diabetic Patients on Follow-Up at University of Gondar Hospital, Northwest Ethiopia. Advances In Nursing. 2016: 1-7 Article ID: 8579157

16. Bagonza J, Rutebemberwa E, Bazeyo W. Adherence to anti-diabetic medication among patients with diabetes in eastern Uganda; a cross sectional study. Biomedical Care Health Services Reseacrh. 2015;15:168

17. Claxton AJ, Cramer J, Pierce C. A systematic review of the associations between dose regimens and medication compliance. Clinical Therapy. 2001;23:1296-310.

18. Kirman MS, Rowan-Martin MT, Levin R,Fonseca VA, Schmittdiel JA, Herman WH et al. Determinants of Adherence to Diabetes Medications: Findings From a Large Pharmacy Claims Database. Diabetes Care. 2015; 38:604-609

19. Balkhi B, Alwhaibi M, Alqahtani N, Alhawassi T, Alshammari TM, Mahmoud $\mathrm{M}$ et al. Oral antidiabetic medication adherence and glycaemic control among patients with type 2 diabetes mellitus: a cross-sectional retrospective study in a tertiary hospital in Saudi Arabia. Biomedical Journal Open. 2019;9:e029280.

20. LeeCS,TanJHM,SankariU,EileenKohYL, Chuan Tan N. Assessing oral medication adherence among patients with type 2 diabetes mellitus treated with polytherapy in a developed Asian community: a cross sectional study. Biomedical Journal Open. 2017;7:e016317. 